\title{
Based on Hybrid Particle Swarm Optimization Algorithm Respectively Research on Multiprocessor Task Scheduling
}

\author{
Tian Hui ${ }^{1, a}$ \\ ${ }^{1}$ Guilin University of Technology, Guangxi 541006, China \\ a781144756@qq.com
}

\begin{abstract}
Keywords: Multiprocessor, Task scheduling, TSP, HPSO.
Abstract. Multiprocessor system plays an important role in the computer, in order to improve the parallel computing performance of the system, its essence is to solve a multiprocessor system task scheduling algorithm of NP problem, and the TSP (traveling salesman problem) is a typical NP-complete problem. This article will be attributed to solve multiprocessor task scheduling multiprocessor task scheduling of TSP combination optimization problems. In this article, through the experiment to verify the hybrid particle swarm optimization algorithm and genetic algorithm in solving TSP multiprocessor task scheduling optimization problems, the experimental results show that the hybrid particle swarm optimization algorithm in solving the questions of different size of task scheduling is not only to solve the high quality, and solving the faster, the perform better than genetic algorithm.
\end{abstract}

\section{Introduction}

At present, heterogeneous multi-core processor is the development direction of the processor, and a computer also contains a number of heterogeneous multi-core processors. In a heterogeneous multi-core processor platform, how to assign different tasks to different processors to obtain reasonable running time minimum or maximum output weights is the primary problem of multi-core processor can play the advantages of performance. The task scheduling problem has been proved to be NP-hard problem, unable to obtain optimal solution in polynomial. Therefore, the task scheduling problem in heterogeneous multi-core processors has become the research focus of heterogeneous multi-core technology.In order to verify the proposed hybrid particle swarm algorithm (HPSO) in the task scheduling algorithm for heterogeneous multi-core processor between, and carried out the experiment without loss of generality in the simulation platform of MATLAB, and in comparison with the classical genetic algorithm (GA) are compared and analyzed.

\section{The Task Scheduling Problem Description}

This paper discusses the task scheduling problem in heterogeneous multi-core processors. The task scheduling of heterogeneous multi-core processor system is mainly focused on the task model with constraint, and the constraint relation of DAG expression task is generally used. Assuming a system model with 3 heterogeneous multi-core processors and 14 constrained task relationships, the node of the DAG graph represents the task, which reflects the dependencies between tasks. As shown in figure 1 .

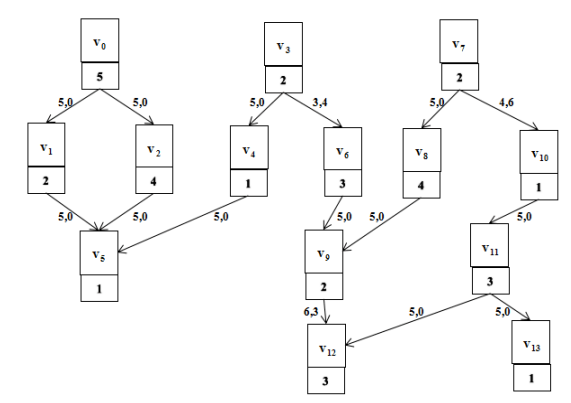

Fig. 1 fourteen tasks DAG case diagram 
A DAG diagram such as the following tasks, task communication between different processors, for the structure of the DAG map using task scheduling algorithms usually execute its key communication overhead between nodes, so the task duplication and eliminate the communication overhead between nodes and tasks.

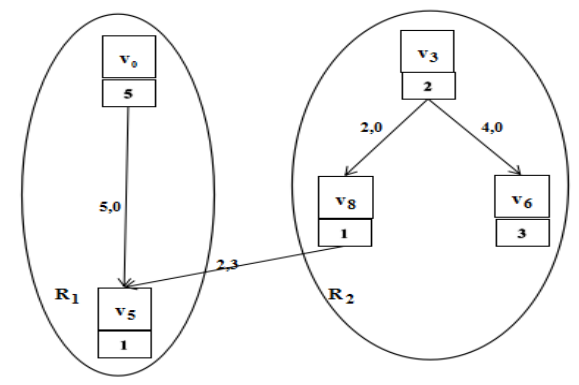

Fig. 2 DAG diagram of communication between different tasks After the task of copying the DAG diagram as follows.

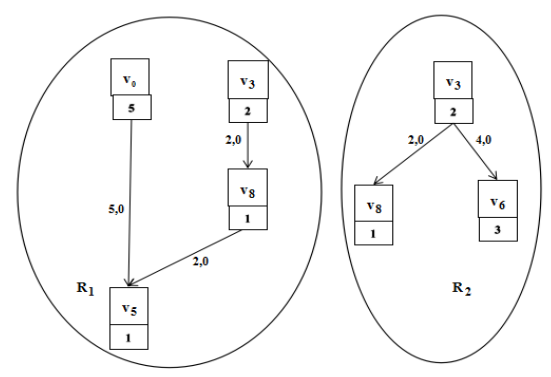

Fig. 3 After the task of copying the DAG map

Therefore, the task allocation scheme is summarized as follows: firstly, in order to reduce the time required for communication between tasks, frequent communication tasks assigned to the same processing nodes; secondly, all belong to the same task must be assigned to the same node, this is the same process share the same the storage unit, and each processing node are independent of each other; finally, to ensure the processing nodes and load balance.

\section{Task Scheduling}

The task scheduling problem has been proved to be NP-hard, the task allocation scheme will appeal to the corresponding task allocation for heterogeneous multi-core processor, the scheduling algorithm proposed in this paper is based on the above after the completion of the task allocation, In this paper, the problem of the combinatorial optimization problem is proposed, and a hybrid particle swarm optimization (PSO) algorithm is proposed to solve the problem when the task size is too large. In this paper, a hybrid particle swarm optimization algorithm is proposed to improve the results of previous studies. The scheduling of the following algorithms is aimed at the task scheduling of independent heterogeneous multi-core processors, and in order to facilitate the understanding, this paper considers that each independent processor is assigned 14 tasks.

Hybrid particle swarm optimization algorithm in TSP group task scheduling implementation of the optimization problem.

(1) Particle the task of individual coding:task of the individual coding using integer coding method, each task was performed tasks, such as the algorithm in this paper assumes that the execution of 12 tasks, each task coding for[4,6,7,10,3,5,11,8,2,9,1,12,13,14 ], said to perform a task from the task 4, 4, 6,.. Eventually return to task 4, so as to complete the execution of all tasks.

(2) the fitness value:Particle fitness value is expressed as the duration of all the tasks, the said fitness $(i)=\sum_{i, j=1}^{n} D_{i, j}$, among n number for task, $\boldsymbol{D}_{i, j}$ said i mission to $\mathrm{j}$ what walked the length . 
(3) the crossover operation:By individuals in the population and individual the extreme value and the extreme value in the group to cross to get updates, cross method USES the same USES the integer crossing method. Choose two cross position first, secondly the the extreme value of the individual and the individual or the extreme value of individual and group crossed, assume that cross location selected for 10 and 12, the crossover operation is: Individual[4,6,7,10,3,5,11,8,2,9,1,12,13,14 ] and the extreme value $[4,2,8,10,3,5,11,7,6,9,1,12,13,14]$ for individual new crossover operation process for the new, the new individual for: $[4,6,8,10,3,5,11,8,2,9,1,12,13,14]$, at the same time with repeated coding on the whole, the individual in the adjustment of $[4,6,8,10,3,5,11,7,2,9,1,12,13,14]$, and then keep excellent individual strategy, only when the new particle fitness value is higher than the old particles to select update.

(4) mutation:The method uses two two internal position of individual exchange principle, first randomly determined two positions, such as positions 8 and 2, then swap, variation process:the $[4,6,7,10,3,5,11,8,2,9,1,12,13,14]$ variant $[4,6,7,10,3,5,11,2,8,9,1,12,13,14]$, and still retain the best individual strategy, only when the new particle fitness value is higher than the old when they choose to update particle.

\section{Simulation Experiments and the Results Analysis}

The test shows that the genetic algorithm and hybrid particle swarm algorithm to parallel computing on multiple processors, but the hybrid particle swarm algorithm in the number of tasks executed regardless of time, he performance experiment of task scheduling algorithm on MATLAB.

(1) when the number of threads is $\mathrm{N}=14$, the number of population is about 100 , and the iteration algebra is about 200 . The results of the standard burma14 position coordinate simulation of the task are as follows:

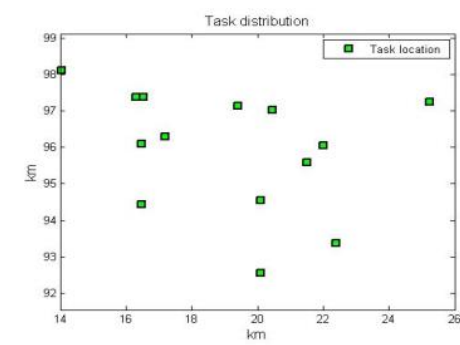

Fig. 4 Task distribution graph when task $\mathrm{N}=14$
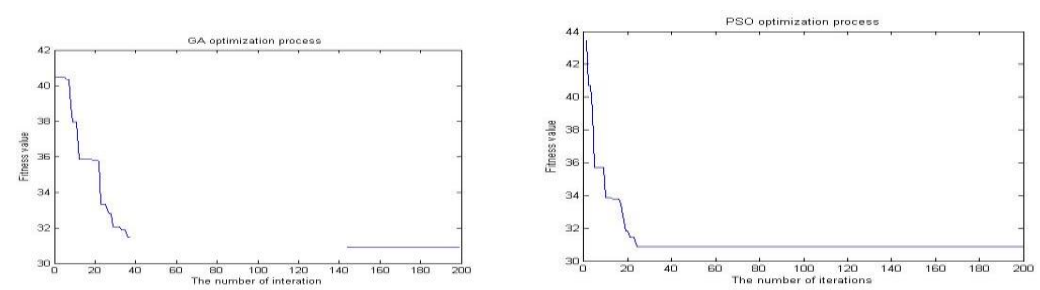

Fig. 5 The comparison of GA and HPSO in the process of optimizing the number of tasks

$$
\mathrm{N}=14
$$

(2) When the number of tasks is $\mathrm{N}=50$, the number of population is 100 , and the iterative algebra is 1000 :
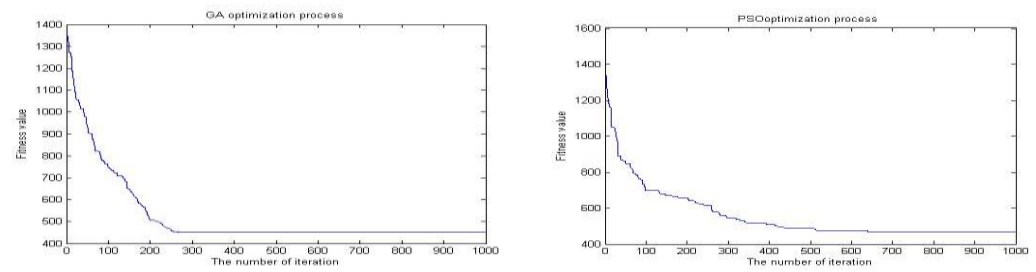

Fig. 6 The comparison of GA and HPSO in the process of optimizing the number of tasks $\mathrm{N}=50$ 
(3) When the number of tasks is $\mathrm{N}=130$, the number of population is 100 , and the iterative algebra is 3000 . The results of the two algorithms are as follows:
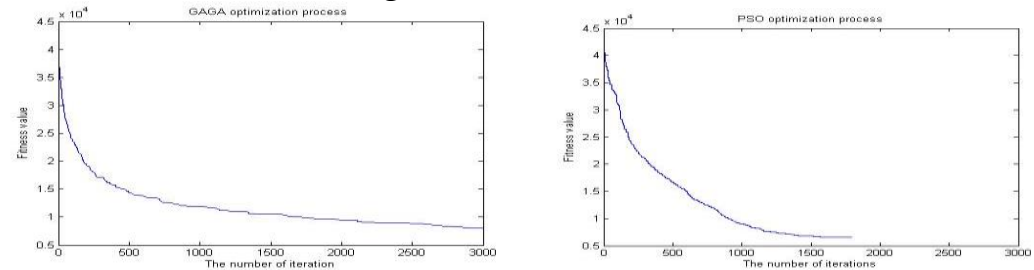

Fig. 7 The comparison of GA and HPSO in the process of optimizing the number of tasks

$$
\mathrm{N}=130
$$

when the $\mathrm{N}=14$ task, as can be seen from figure 7 when the iterative GA algorithm in time to the 100 generation of stable, and HPSO to the 40 generation of the time when the iteration is stable.According to the above data, we can see that when the task $\mathrm{N}=14$, the HPSO algorithm is superior to the GA algorithm in both the optimal solution and the worst solution, the scheduling time, and the HPSO algorithm is very stable.

when the $\mathrm{N}=50$ task, as can be seen from figure 8 when the iterative GA algorithm in time to the 730 generation was stabilized, and HPSO to the 600 generation of the time when the iteration has been stable.According to the above data, we can see that when the task N=50, the HPSO algorithm is superior to the GA algorithm in both the optimal solution and the worst solution, the scheduling time, and the HPSO algorithm is very stable.

when the $\mathrm{N}=130$ task, as can be seen from figure 9 when the iterative GA algorithm in time to the 3000 generation did not begin to stabilize, and HPSO to the 1700 generation of the time when the iteration has been stable.Through the above data can be obtained when the $\mathrm{N}=130$ task, the GA algorithm has not scheduling and processing tasks, while the HPSO algorithm can find the accurate values, so when large amount of tasks, the HPSO algorithm can reasonable scheduling of tasks.

\section{Summary}

In order to improve the efficiency and performance of task scheduling in multiprocessor systems. In this paper, the genetic algorithm and hybrid particle swarm optimization algorithm respectively in combination of TSP solving multiprocessor task scheduling optimization problem was studied. By comparing the results with genetic algorithm, the solution quality of hybrid particle swarm algorithm is higher and faster execution speed, can effectively shorten the task completion time, and improve the performance of multiprocessor system.

\section{References}

[1] Gupta S, Agarwal G, Kumar V. Task scheduling in multi-processor system using genetic algorithm [C] / /Proc of the 2nd International Conference on Machine Learning and Computing. [S. 1. ] : IEEE Press, 2012: 267-271.

[2] Yang Huihua, Zhang Xiaofeng. Multiprocessor cuckoo search based on Ren Service scheduling algorithm [J]. computer science.2015.1.

[3] Yuan Yun. Task scheduling in many core parallel system based on[J]. computer applications, 2011,12.

[4] Ma Yongjie, [J]. cloud Wen Xia genetic algorithm to calculate the research progress.Computer application research, 2012,29 (4): 1201-1206.

[5] Jia Jianfang, Yang Ruifeng, Wang Li. Hybrid genetic particle swarm optimization research on the algorithm of J. Automation instrument, 2013, 34 (9). 